\title{
On the staking of miniaturized air-breathing microbial fuel cells
}

S. Mateo ${ }^{1}$, A. Cantone ${ }^{1,2}$, P. Cañizares ${ }^{1}$, F. J. Fernández-Morales ${ }^{1}$, O. Scialdone ${ }^{2}$, M.A. Rodrigo $^{1, *}$

${ }^{1}$ University of Castilla-La Mancha, Faculty of Chemical Sciences \& Technologies, Chemical Engineering Department, Avenida Camilo José Cela, 12. 13071 Ciudad Real, Spain.

${ }^{2}$ Università degli Studi di Palermo, Dipartimento dell'innovazione industriale e digitale, Ingegneria Chimica, Gestionale, Informatica, Meccanica, Viale delle Scienze, Palermo, Italy

*Author to whom all correspondence should be addressed: manuel.rodrigo@uclm.es 


\begin{abstract}
This work focuses on the scale-up of the MFCs by miniaturization and multiplication strategy. Performances of five stacks containing 1, 2, 5, 8 and 16 MFCs are compared. Each stack was evaluated under individual, parallel and series electrical connection as well as for cascade or individual hydraulic connection. Cascade feeding mode with a tank per stack favours the COD removal when the number of MFCs in the stack increases. However, despite operating without COD limitations, the energy production was disadvantaged. By changing the feeding system of a tank per stack into an individual tank per MFC, the performance of the whole stack enhances considerably. Stacking in series can increase the voltage 6 times while stacking in parallel can increase the current output 4.2 times. For example, $8 \mathrm{MFCs}$ can achieve $2.03 \mathrm{~V}$ connected in series and $6.98 \mathrm{~mA}$ connected in parallel. In addition, the power can be increased up to 9.66 times.
\end{abstract}

\title{
Keywords
}

Microbial fuel cell; stack; cascade; miniaturization; multiplication.

\section{Highlights}

- The higher the number of MFC in a stack, the higher the COD consumption

- Electricity production is limited with the number of MFCs in the stack

- Individual feeding tanks per MFC avoids electricity generation limitations

- Series connection increases up to 6 times the voltage in the stack of 8 MFCs

- Parallel connection increases the current up to 4.2 times in the stack of 8 MFCs

\section{INTRODUCTION}


Microbial fuel cells (MFCs) can help to recover part of the energy contained in wastewater, becoming a renewable energy source alternative (Logan, 2008). Unfortunately, its practical application is limited because of the low power generated (50$200 \mathrm{~W} \mathrm{~m}^{-3}$ ) and low working potentials (in a range of 0.3-0.5 V) achieved by single MFCs. For this reason, scale-up of MFCs has received increasing attention during last years and a great scientific effort has been focused on methodologies to get energy dense devices.

Scale-up by increasing the size of the MFC does not result in a proportional increase of the output energy and the efficiency of the system, mainly because of the associated increase of the internal resistance (Dekker et al., 2009) and the reduction of the electrode surface to volume ratio (Mateo et al., 2015b). However, small MFCs result in higher energy recovery efficiencies. By miniaturing the MFC, shorting the electrodes spacing, increasing the surface-volume ratio and reducing the volume of the chambers, the typical parameters that influence the MFC performance (operational conditions and design parameters) could be better controlled. Hence, the energy recovery could be enhanced by multiplying small MFCs leading to a new proposal of scaling-up, which is called 'miniaturization and multiplication of MFCs' (Chouler et al., 2016; Ieropoulos et al., 2010; Ieropoulos et al.). The miniaturization consists of reducing considerably the size of the MFC until building micro-chambers (Choi, 2015; Papaharalabos et al., 2015; Qian \& Morse, 2011; Ringeisen et al., 2006). On the one hand, the miniaturization reduces the volume of the chambers as well as the electrode spacing, at the same time that increases the surface-volume ratio. The combination of these factors allow to maximizes the feed flows into the biofilm, making easier the electron transference to the anode and the pathway of the protons from the anode to the cathode, lowering internal resistance and therefore improving the MFC efficiency (Ringeisen et al., 2005). As an example of laboratory scale-MFCs, a reported study shows that a projected anodic surface area of 2 
$\mathrm{cm}^{2}$ resulted in an impressive power of $3000 \mathrm{~mW} \mathrm{~m}^{-2}$ (Ringeisen et al., 2006; Ringeisen et al., 2007), whereas in another work with a projected anodic surface area much larger, $232 \mathrm{~cm}^{2}$, the maximum power density reached only $26 \mathrm{~mW} \mathrm{~m}^{-2}$ (Liu et al., 2004). Concerning to the volume, Walter et al. showed that a MFCs operated with volumes of 6.3 or $29.6 \mathrm{~mL}$ resulted in a power density of 0.44 and $0.38 \mathrm{~W} \mathrm{~m}^{-3}$, respectively (Walter et al., 2016b). On the other hand, the multiplication of MFC conforming stacks as scaleup strategy avoid energy losses ensuring at the same time high efficiencies (Dekker et al., 2009; Ieropoulos et al., 2008; Ieropoulos et al., 2013a; Walter et al., 2016b). This process is named multiplication (Chouler et al., 2016).

It is very important to multiply devices with very simply structure. Indeed, air-breathing MFCs are easier to arrange in a stack than double chambered MFCs and requires less space. Furthermore, air-cathodes guarantees no limitations in the availability of final electron acceptor (Mateo et al., 2015a). For practical applications, the output energy can be increased by connecting electrically each single MFC assembled in the stack (Chouler et al., 2016; Ieropoulos et al., 2010). Series connections lead to a potential equal to the sum of individual ones while connecting in parallel increases the current output up to the sum of the currents exerted by each MFC (Dekker et al., 2009; Ieropoulos et al., 2013b). However, these connections can produce contact potential losses and potential reversal due to imbalanced potentials caused by different reactions kinetics on the electrodes leading to erroneous operations, which worsen considerably the performances of the stack (Zhang \& Angelidaki, 2012; Zhao et al., 2017). Zhao et al. developed a self-stacked submersible MFC, which degraded glycerol, obtaining the maximum current in parallel (5.7 $\mathrm{mA})$ and the maximum potential in series (1.21 V) (Zhao et al., 2017). The maximum power density exerted was similar in both cases, around $450 \mathrm{~mW} \mathrm{~m}^{-2}$ (Zhao et al., 2017). Chouler et al. achieved 0.063 and $0.243 \mathrm{~W} \mathrm{~m}^{-3}$ arranging 3 MFCs in series and in parallel, 
respectively, while the power density reached by one unit was $0.053 \mathrm{~W} \mathrm{~m}^{-3}$ (Chouler et al., 2016).

In addition to the electrical advantages obtained by the scaling up of MFC, the wastewater treatment capacity of these bio-electrogenic devices can be enhanced by connecting hydraulically each individual MFCs of the stack (Ledezma et al., 2013), which results in a cascade of MFCs where the outlet of the first MFC is the inlet of the second MFC and so on until the last MFC. The cascade reduces the bulk volume do not exposed to the electrodic surface area increasing therefore the hydraulic retention time in the nearby of the electrodes (Walter et al., 2016a). In addition, it allows shorter diffusion distance and a more efficient volumetric electrode performance (Walter et al., 2016a). For example, Monasterio et al. achieved a further algal removal employing a cascade of 3D printed miniature air-cathode MFCs (Monasterio et al., 2015). However, cascades of a large length could result in mass transfer limitations in the latter stages due to the operation at substrate concentrations near and even lower than the half saturation coefficient. This problem can be avoided by increasing the flow rate, which increases the loading rate of the system and the turbulence leading to a more uniform distribution of the substrate, but increases the operational costs at the same time that could lead to leakages due to the higher operational pressure. Hence, scale up by miniaturization and multiplication seems to be a good choice for improving the performance of MFC, but further work has to be carried out in order to shed light on the way to optimize these devices. In addition, despite the number of publications related to stacking MFCs, most of them tested different electrical and hydraulic connections for a unique stack (Walter et al., 2016b; Winfield et al., 2011; Zhao et al., 2017). However, the main aim of this work is to evaluate the effect of electrical and hydraulic connections in a stack as well as the influence of increasing the number of MFCs configuring a stack over its performance. For this reason, in this work five devices with different numbers of stacked MFCs (ranging from 1 to 16) were 
build up. The electrical connection (series or parallel) as well as the hydraulic connection (individual feeding or cascade) were studied in order to understand and deep into the stacking process with the number of MFCs. 


\section{MATERIALS AND METHODS}

Experimental set-up. All the MFCs were build up with the same characteristics, size and materials. Each MFC consisted of a cathode opened to the atmosphere, which employed oxygen gas as final electron acceptor. The cathode was based on carbon paper (Freudenberg C2) with a $10 \%$ of Teflon and a load of $0.5 \mathrm{mg} \mathrm{Pt} \mathrm{cm}^{-2}$ on its surface (Mateo et al., 2017b). The face of the cathode not exposed to the air faced a Nafion N117 proton exchange membrane (DuPontTM Nafion PFSA Membrane). This membrane separated the anodic and the cathodic chambers. The anodic chamber had a free volume for the wastewater of $0.346 \mathrm{~cm}^{3}$ and contained carbon felt as anode made of. Both electrodes had an active area of $0.866 \mathrm{~cm}^{2}$ and were connected externally by a resistance of $120 \Omega$. The distance of the electrodes and the membrane was reduced as much as possible to avoid high internal resistances. These individual MFC were used to configure stacks containing 1, 2, 5, 8 and 16 units and named S-1MFC, S-2MFCs, S-5MFCs, S-8MFCs and S16MFCs respectively. A detailed scheme of the stack configurations is presented in Figure 1.

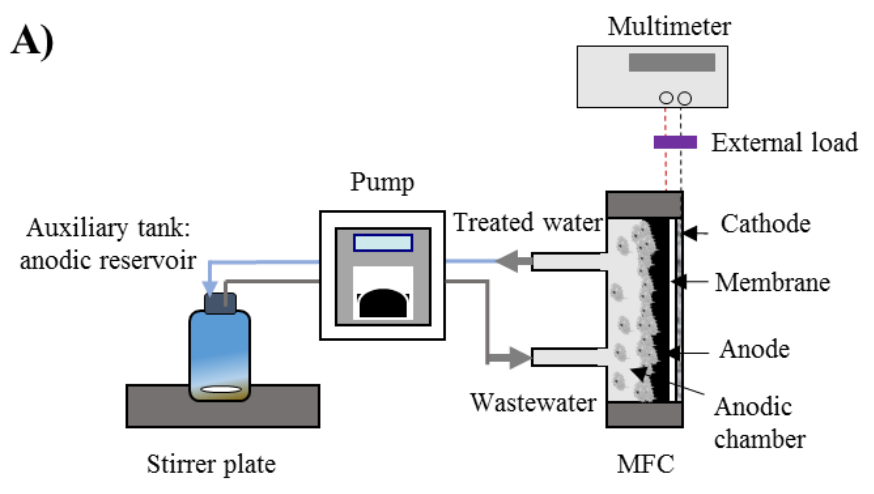

B)
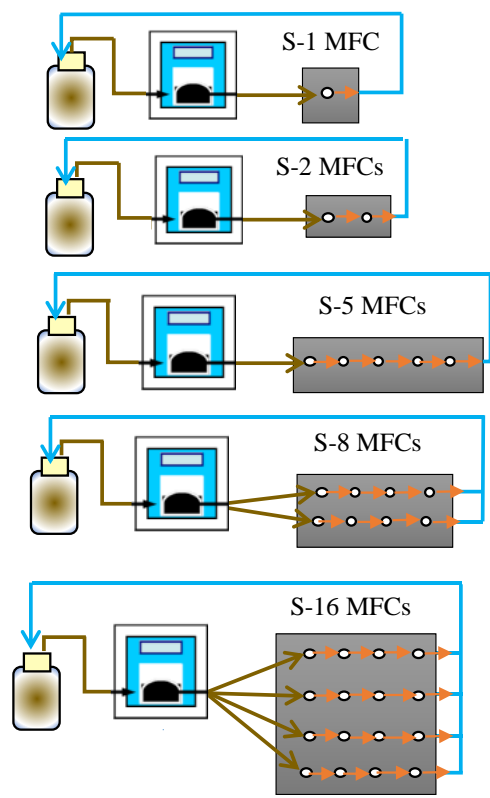
Figure 1. A) Detailed scheme of the experimental set-up for S-1MFC. B) Scheme of the experimental set-up for the stacks operated.

\section{Inoculation and operational procedure.}

The MFCs were inoculated with activated sludge obtained from the Wastewater Treatment Plant of Ciudad Real in Spain. More information about this facility can be found elsewhere (Rodríguez Mayor et al., 2004). This sludge underwent sedimentation to increase its biomass concentration. Once the process was finished, $50 \% \mathrm{v} / \mathrm{v}$ of the decanted sludge and $50 \% \mathrm{v} / \mathrm{v}$ of the supernatant (wastewater) was used to fill each auxiliary anodic reservoir tank. It was recirculated through the system for 24 hours. Then, during the second and third days, $50 \%$ of the volume was removed and replaced by fresh sludge following the same operational pattern of the first day. At the fourth day and during the rest of the experiment, a sludge age of 2.5 days was established as operational parameter. This sludge age has been reported as the optimal sludge age to develop a bioelectrogenic consortia in the anodic chamber (D'Angelo et al., 2017). Thus, $46 \mathrm{~mL}$ of the auxiliary anodic tank was daily purged from the system and replaced by fresh synthetic wastewater.

Hydraulic connection. Figure 1.a shows the scheme of the operation mode for a single MFC. A similar scheme can be used for the rest of the stacks by stacking horizontally several MFC as shown in Fig 1.b. In all the stacks, a reservoir tank of $115 \mathrm{~mL}$, containing the inoculum and the wastewater (which is used as the fuel of the MFC), was connected to each stack. The hydraulic connection between the individual MFCs conforming the stacks is shown in Fig. 1b. For the stacks of 1, 2 and 5 MFCs, the MFCs were connected in horizontal cascade. Thus, for example, in the stack of 2 units the outlet of the first MFC was the inlet of the second MFC. In the case of the stacks of 8 and 16 MFCs, where 
several rows of 4 MFCs were necessary, only horizontal cascades were made so the outlet of every cascade of 4 MFCs were recirculated to the reservoir tank.

Electrical connection. During the start-up, each MFC was connected to an external resistance of $120 \Omega$ individually. For the electrical connection study, parallel and series connection were tested. In parallel connection, all the anodes of the MFCs making up the stack were connected, all the cathodes were also connected resulting in two outputs, one for the anode and one for the cathode closed by an external load of $120 \Omega$. When connecting in series, the cathode of the first MFC was connected to the anode of the second MFC. Finally, the anode and the cathode that remained unconnected were linked by a $120 \Omega$ external resistance.

Synthetic wastewater and Analytical methods. Synthetic wastewater was used to avoid fluctuations in its composition. Working in this way, it is favored the reliability, reproducibility and comparability of the results. Before its use, the synthetic wastewater was subjected to a sterilization process in an autoclave at $120^{\circ} \mathrm{C}$ for 15 minutes according to the literature (Fernandez-Morales et al., 2010). The composition of the synthetic wastewater was $16.10 \mathrm{~g} \mathrm{~L}^{-1}$ of sodium acetate, $2.77 \mathrm{~g} \mathrm{~L}^{-1}$ of sodium carbonate, $1.11 \mathrm{~g} \mathrm{~L}^{-1}$ of potassium dihydrogen phosphate, $1.25 \mathrm{~g} \mathrm{~L}^{-1}$ of calcium chloride, $0.92 \mathrm{~g} \mathrm{~L}^{-1}$ of hexahydrate magnesium chloride, $1.85 \mathrm{~g} \mathrm{~L}^{-1}$ of ammonium sulfate and $0.07 \mathrm{~g} \mathrm{~L}^{-1}$ of ferric ammonium sulfate. All the reagents were of analytical grade.

The determination of the Chemical Oxygen Demand (COD) consumption by the stacks (fuel consumed) was monitored in terms of soluble COD removal from the system. Before the measurement, samples were filtered by using Nylon filters, $0.45 \mu \mathrm{m}$ pore size, to avoid COD interferences due to the presence of microorganisms in the liquid bulk. COD analysis was carried out according to the Standard 2540 D through colorimetry (Federation \& Association, 2005; Water Environment et al., 1905). Soluble COD removal 
was determined as the difference between the concentration at the beginning and the end of a daily cycle.

Electrochemical characterization. The electricity generation under close circuit conditions, circuit close with the external load, was continuously monitored through the automatically measurement of the exerted potential with a Keithley 2000 Multimeter. Polarization curves were obtained with a potentiostat/galvanostat AUTOLAB PGSTAT30 at a scan rate of $0.001 \mathrm{~V} \mathrm{~s}^{-1}$. Before carrying out this technique, the system were left in open circuit conditions for two hours in order to obtain accurate Open Circuit Voltage (OCV) measurements (Mateo et al., 2016). 


\section{RESULTS AND DISCUSSION}

\section{Effect of the number of cells on the performances of the stack}

Before studying the behavior of the stacks, the electrical performance of each individual MFC was evaluated. After studying individually the 32 MFCs used it was observed that the the standard deviation of the voltages and currents exerted were lower than $10 \%$. Once validated the reproducibility of the MFCs, the performance of the stacks was studied. First trials of experiments were carried out by connecting separately each MFC from an electrically point of view. Fig. 2 shows the daily current density exerted by the stack of 2 MFCs. In this figure, it can be observed the three typical phases of bacterial growth: lag, exponential and stationary phases. During the first three weeks, corresponding to the lag phase, there was any electricity production. Then, from $24^{\text {th }}$ day, a noteworthy increase in the electricity production was observed in both cells, which correspond to the exponential phase. Finally, the steady state was reached after 57 days of operation. From this moment the system can be considered to be operated under stationary conditions.

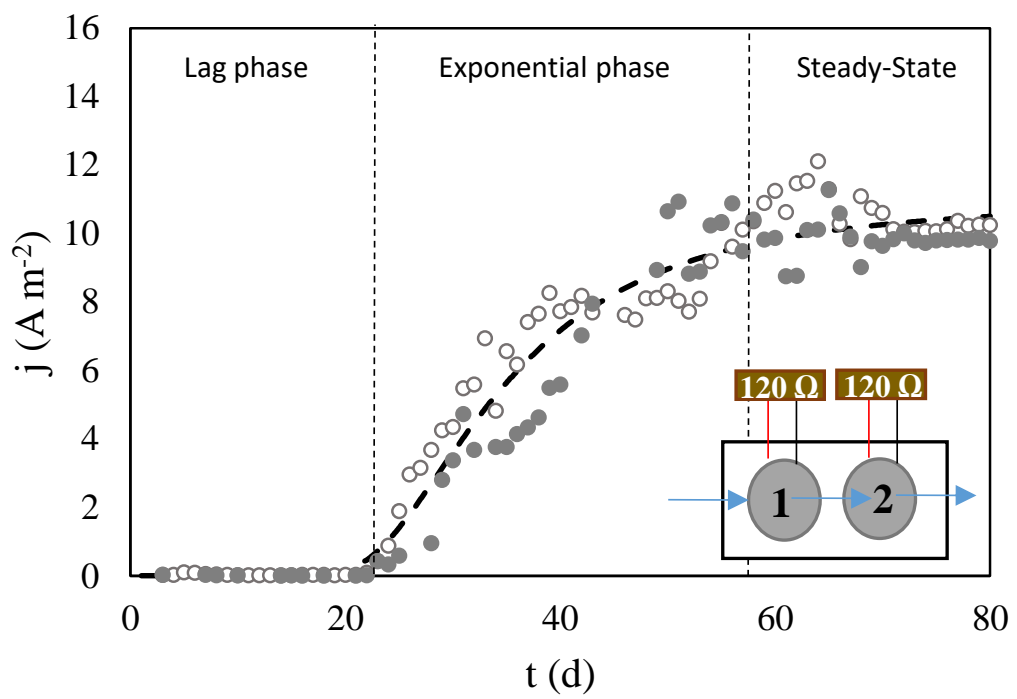

Figure 2. Time course of the exerted current density by the MFCs of the stack of 2 MFCs fed in cascade. (०) First MFC; $(\bullet)$ Second MFC. 
As can be seen in figure 2, the performance of each MFC conforming the stack of 2 MFCs was very similar. In order to study the influence of the number of MFC in a stack over the start-up stage, the length of the lag phase as well as the time required to reach the steady state were registered and are presented in Figure 3. In this figure the performance of all the stacks were studied paying special attention to the time required to start producing energy and the exerted current density. As indicated above, each single MFC was operated under a load of $120 \Omega$ and the time required by each single MFCs was measured and the average value for each stack calculated. As it can be observed in Fig. 3 , longer operation times are required for the larger stacks even if the data are not very different. To understand this trend, it is worth to mention that there was an auxiliary tank of $115 \mathrm{~mL}$ per stack. In this sense, the higher the number of MFC in the stack, the higher the electrode surface and therefore the surface available for biofilm growth with the same volume of initial inoculum in each stack. Thus, a higher surface to be covered by bacteria requires a longer time to reach the steady-state.

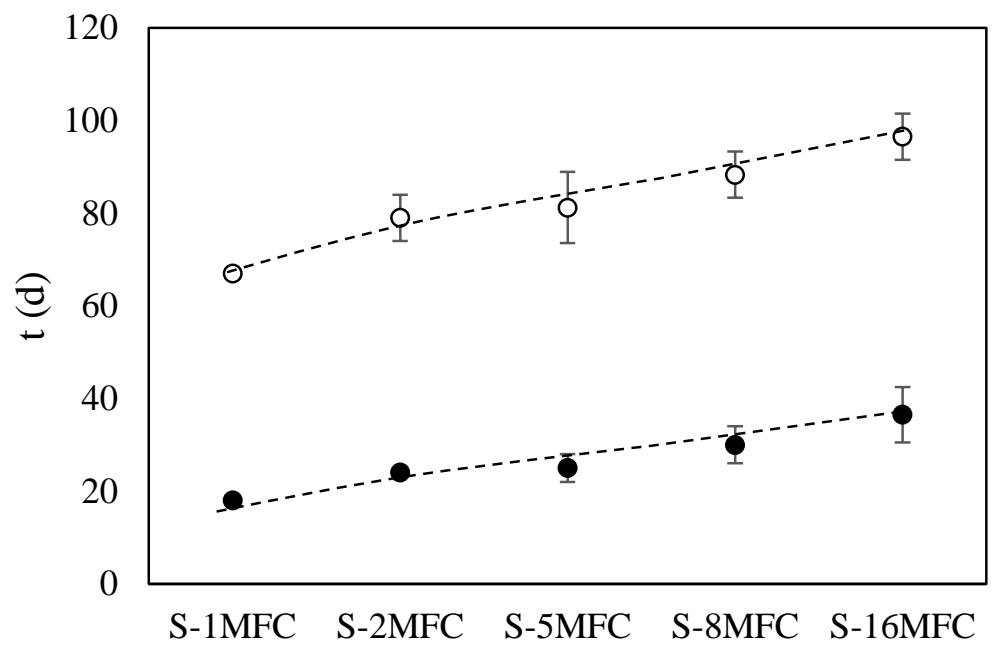

Stack 
Figure 3. Average time to start producing electricity $(\bullet)$ and to achieve the steady state (value of the current density stabilized within the range $\left.\pm 1.0 \mathrm{~A} \mathrm{~m}^{-2}\right)(0)$ by each stack. Data is the average of the single MFCs of each stack and error bars represented their STDV.

With regard to the suspended biological culture, a different behavior was observed. The total suspended solids (TSS) is a parameter describing the concentration of microorganisms in the recirculating tank of the microbial fuel cell (Mateo et al.). As shown in Fig. 4, for all adopted stacks, there was a decrease in the TSS concentration down to approximately the same steady state value, about $1000 \mathrm{mg} \mathrm{L}^{-1}$. The similar behavior in all the cases can be explained taking into account that the five devices were seed with the same volume of inoculum and operated under the same sludge age (Mateo et al., 2017a; Mateo et al.). Hence, a similar production of suspended microorganisms can be expected. This decrease of the TSS concentration with time to a constant value indicates that, during the acclimation of the biological culture, not all microorganisms seed were able to survive. Additionally, a fraction of sludge is daily removed due to the purge required to stablish the required sludge age (Vicari et al., 2017). It is worth to mention that the stacks started to produce electricity approximately when the TSS concentration reached a constant value, pointing out that the generation of electric energy requires the growth of the electrogenic population and the removal of the competing ones. 


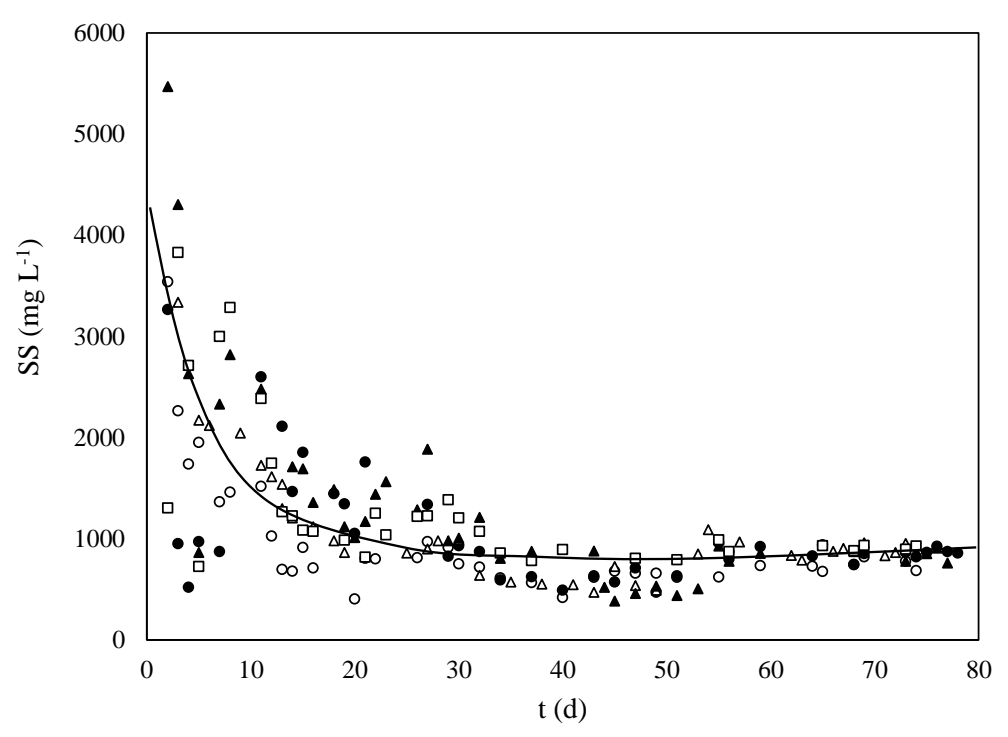

Figure 4. Evolution of the suspended solids in the anodic reservoir per stack. (०) Stack

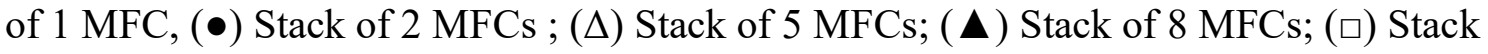
of 16 MFCs.

With regard to the COD removal, it was observed that, the larger the number of cells in the stack, the higher the COD removal (Fig.5a). As the concentration of suspended solids was very similar in all the feeding tanks regardless the number of MFCs stacked, the total increase of the COD removal for the larger stacks could be directly related to the larger biofilm attached to the anodic electrode due to the higher electrodic surface. However, attending to fig $5 \mathrm{~b}$, it can be observed that larger stacks results in lower current output per MFC. This fact indicates that the increase in the COD removal in the larger stacks can only be explained because of the combination of the biofilm and suspended microorganisms metabolisms. In this sense a different metabolisms was identified as reflects the evolution of the $\mathrm{pH}$ values: 7.12 for $\mathrm{S}-1 \mathrm{MFC}, 6.98$ for $\mathrm{S}-2 \mathrm{MFCs}, 6.95$ for S5MFCs, 6.71 for S-8MFCs and 6.36 for S-16MFCs. It is important to mention that the $\mathrm{pH}$ of the synthetic wastewater added on a daily basis was 7.45. The decrease of the $\mathrm{pH}$ with the number of MFCs stacked suggest the coexistence of an acidogenic population 
excreting acids as result of its metabolic activities. This reduction in the $\mathrm{pH}$ could be the responsible of the lower electrogenic specific performance when operating with high number of MFC in the stack.

B)
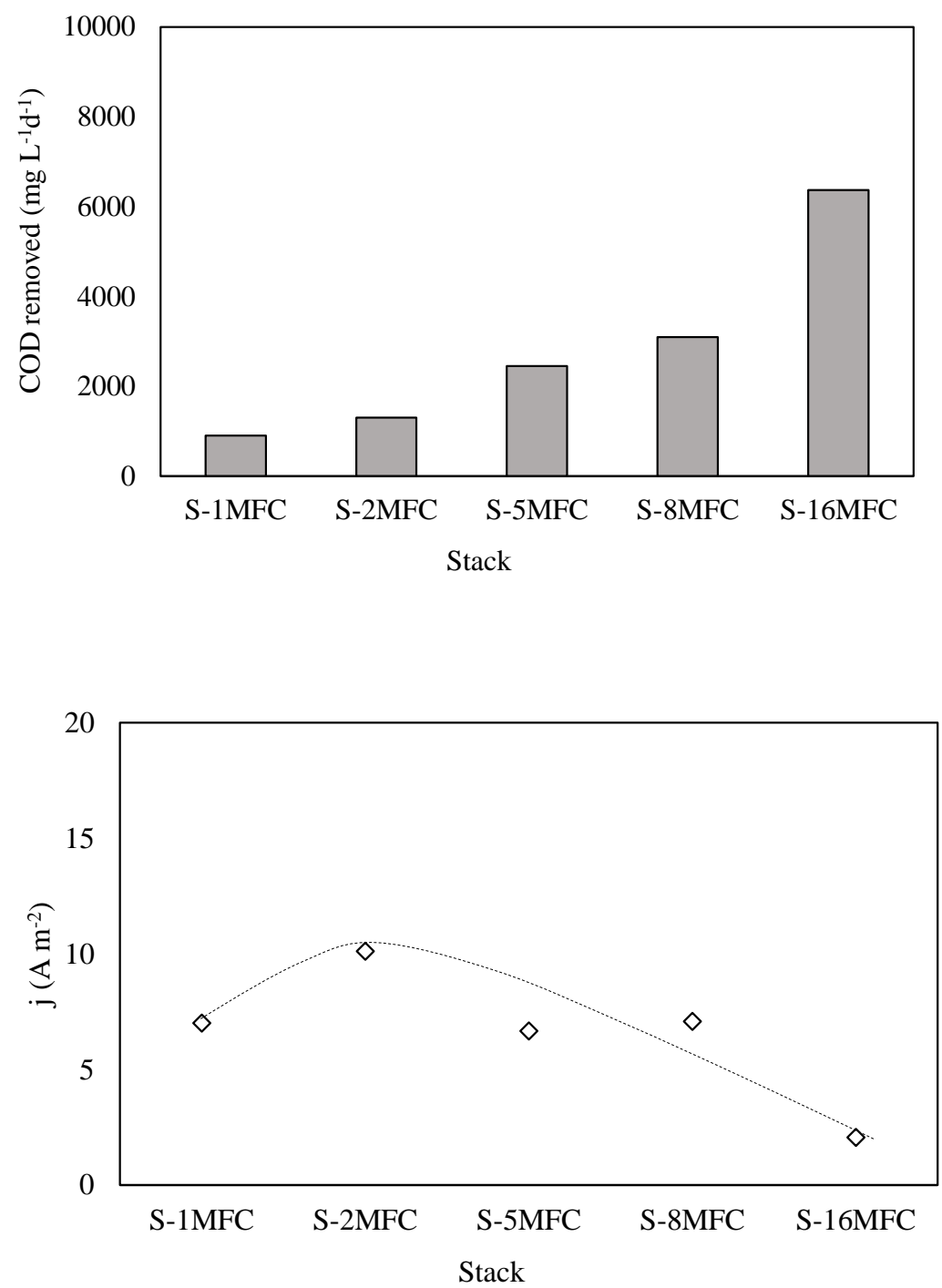

Figure 5. a) Evolution of the COD removed daily, under steady-state conditions, for the entire stack. b) Average current density exerted daily by the individual MFCs of each stack.

With regard to the average current density exerted by each individual MFC (fig. 5b), a maximum in the production of electricity was observed for the stack with two cells. In order to explain this result, it has to be taken into account that the larger the number of cells contained in the stack, the higher is the dispersion in the current output of single 
MFCs. For example, the current output for the single cells of S-2MFCs is around $10 \mathrm{~A}$ $\mathrm{m}^{-2}$ while for S-16MFCs, it ranges between $0.01-8.15 \mathrm{~A} \mathrm{~m}^{-2}$. The oscillation in the output signal was also observed by Winfield et al., who operated a stack of 7 MFCs hydraulically connected in series (Winfield et al., 2012). It is important to take into account that the COD concentration is not limiting the system as fig 5a showed that the COD was in excess in all the cases. Therefore, it is worth to consider the hydraulic connection between single cells in the stack: the effluent from each cell goes to the following cell up to groups of four of five cells (larger hydraulic connections were not useful because of the significant increase in the pressure inside the first cells). This means that the fuel fed to each cell depends on its position in the stack, on the number of cells stacked and on the number of hydraulic lines connected to the same reservoir tank. Fig. 6 shows the current density exerted by each cell of the five stacks depending on their "hydraulic position in the stack". It can be observed that the current output increases with the distance to the inlet of the stacks and the dispersion in the data increases with the number of cells stacked. For the smaller devices, results are very similar while for the largest stacks, the current output oscillates considerably. This similar trend for all the stacks makes unnecessary replicates. In addition, the use of a sole reservoir tank per stack may contribute to the poor electrical performance. Each tank was seed with the same volume of initial sludge and therefore the larger the stack, the higher the number of MFCs to distribute the inoculum of sludge. Thus, when stacking MFCs, which are fed with the same reservoir, the possibilities of low performance of single cells increased with the number of cells arranged in the stack leading to a worse global performance mainly due to the lower ratio microbial seed to anodic volume. This fact clearly advices against the connection of a very large number of cells in a stack when seeding from a single reservoir tank. 
a)

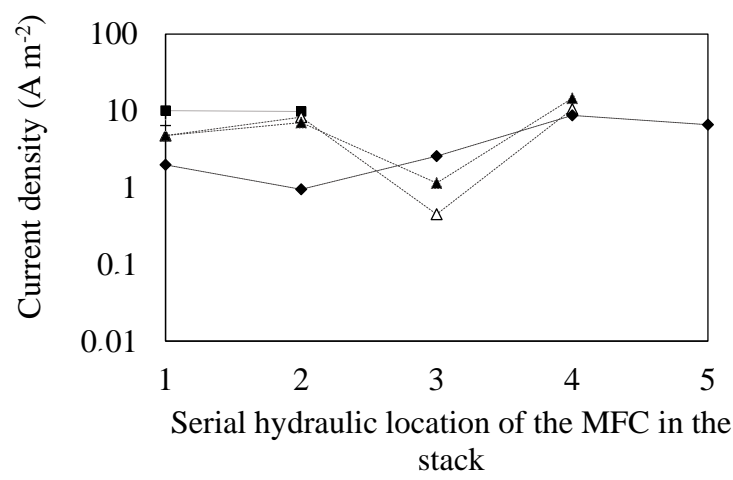

b)

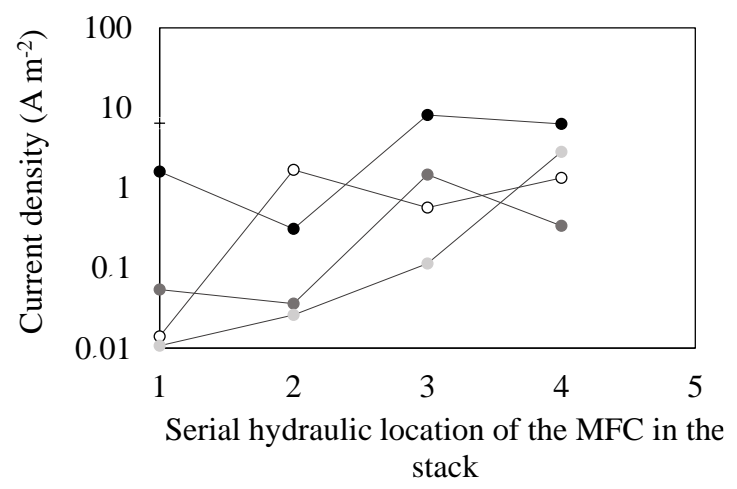

Figure 6. Current density produced by the cells contained in the five devices tests. (a) S1MFC (+); S-2MFC (ם); S-5-MFC $(\diamond)$; S-8MFC ( $\triangle$ hydraulic line $1 \Delta$ hydraulic line 2); (b) S-16MFC (O hydraulic line 1, $\odot$ hydraulic line 2, hydraulic line 3, $\odot$ hydraulic line 4).

Focusing on the electrochemical behavior, Fig. 7 shows the main electrochemical parameters: the open circuit voltage $(\mathrm{OCV})$, the maximum current density $\left(\mathrm{J}_{\max }\right)$ and the maximum power density. Polarization curves were carried out during the most relevant periods: start-up (day 4), exponential improvement (day 40) and steady-state (10 days after reaching the steady state).

a)

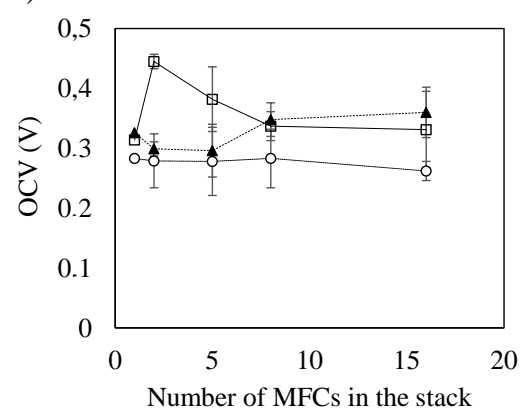

b)

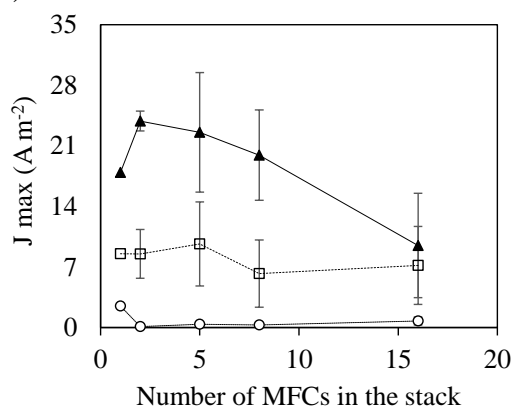

c)

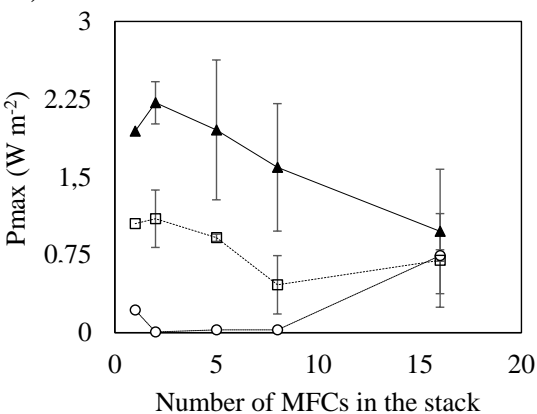

Figure 7. Evolution of the OCV (7a), the maximum current density (7b) and the maximum power density (7c) with the number of MFCs stacked obtained by polarization curves 
recorded during start-up (०), exponential growth phase $(\square)$ and steady state $(\boldsymbol{\Delta})$. Data are an average of the individual values obtained per MFC in each stack.

As shown in fig. 7a, there is an increase in the OCV from the start up to the steady state operation of the cells, which indicates a change in the anodic electrode, most probably due to the formation of the biofilm with electrogenic activity. As can be seen, there was not a great dispersion in the average data obtained for the five stacks tested because in all of them very similar chemical reactions took place. Regarding the maximum current density, changes reflect clearly the formation of the electrogenic biofilm, with a very important increase from the start-up to the steady state, especially for the stacks up to 8 MFCs stacked. Quite high values of the maximum current density were achieved by individual MFCs, up to about $22.6 \mathrm{~A} \mathrm{~m}^{-2}$. Best results were achieved for the stack with 2 MFCs, while the lower current densities were given by the stacks with the large number of cells, as previously observed for measurements performed under close circuit conditions (fig. 5). The same occurs in the evolution of the maximum power density pointing out a poor biofilm quality in larger stacks. These results ratify that the higher the number of MFCs seed with the same volume of inoculum and fed with the same volume of wastewater, the worse the performance of the individual MFCs.

\section{Effect of the electrical connections}

The next step in the research was the evaluation of the influence of the electrical configuration between the MFCs of each stack over the performances of the system. To do that, the individual MFCs of each stack were connected in series and in parallel and the steady state operation was recorded during a week in order to ensure comparable results. Fig. 8 reports the main electrochemical parameters recorded from polarization 
curves for each stack carried out with MFCs connected individually, in series and in parallel.
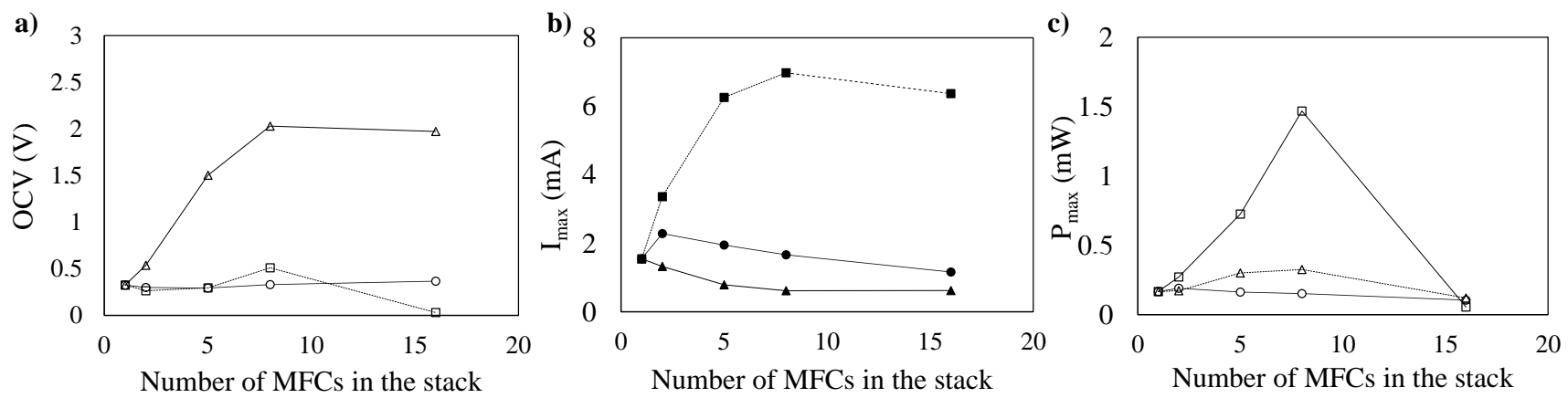

Figure 8. Evolution of the OCV (a), maximum current (b) and maximum power (c) with the number of MFCs stacked individually (०), in parallel ( $\square)$ and in series $(\Delta)$.

It can be observed that an increase in the number of MFCs in the stack results in a considerably increase of the OCV when MFCs are arranged in series and in a rise of the current in parallel. Furthermore, a connection in series leads to a decrease in the current instead of keeping it constant. The same fact is observed for the cell potential when the units are connected in parallel. The maximum power increases with the number of MFCs stacked regardless of the electrical connection. However, it is important to highlight that a number of MFCs in the stack higher than 8 does not improve the performance of the system. This fact can be related to possible enrichment limitations due to the use of a reservoir tank per stack, as it was explained previously, and despite of the larger anodic surface, its bioelectrogenic quality can get better. Therefore, building stacks consisting of more than 8 MFCs would be unpractical unless the feeding operation changes.

These results are very remarkable when comparing with reported works operating stacks connected hydraulically in cascade. In this study, 1000, 694 and $470 \mathrm{~mW} \mathrm{~m}^{-2}$ were achieved when connecting 2, 5 and 8 MFCs in series and 1571, 1676 and $2120 \mathrm{~mW} \mathrm{~m}^{-2}$ when 2, 5 and 8 MFCs were connected in parallel. In the case of stacking 2 MFCs, a 
maximum power density of $9 \mathrm{~mW} \mathrm{~m}^{-2}$ was reported by Kim et al. in series and in parallel (Kim et al., 2017) and $3.88 \mathrm{~mW} \mathrm{~m}^{-2}$ by Oliot et al. (Oliot et al., 2017). Ieropoulos et al. achieved around $60 \mathrm{~mW} \mathrm{~m} \mathrm{~m}^{-2}$ when connecting 4 MFCs regardless the electrical connection (series or parallel) (Ieropoulos et al., 2010) while Walter et al. obtained 144 $\mathrm{mW} \mathrm{m} \mathrm{m}^{-2}$ with 6 MFCs connected in parallel (Walter et al., 2016b).

Another interesting point to be discussed is the COD removal during a daily cycle with the type of electrical connection (Fig. 9). The MFCs were operated in semi continuous mode by feeding the reservoir tank once a day and from that moment, the COD decreases due to its consumption by microorganisms. As it can be observed, this consumption is not only influenced by the number of cells stacked, but also by the electrical connection. As above mentioned for experiments performed with individual electrical connection, the larger the number of cells stacked, the higher the COD consumption, because of the larger electrode surfaces which involves a larger number of microorganism fixed to the anode.

As shown in Fig. 9, where the COD removal trends are compared, the COD removal increases as increases the number of cells in the stack. Additionally, for all adopted stacks, the COD consumption depended on the electrically connection and increased with the following order: individual, serial and parallel. It is important to remind that in every an external load of $120 \Omega$ was used to close the single external circuit as well as the parallel and series circuits. Therefore, in series and parallel connection, the equivalent external load per MFC decreased when increasing the number of MFCs in the stack: $60 \Omega$ for S2MFCs, $24 \Omega$ for S-5MFCs, $15 \Omega$ for S-8MFCs and $7.5 \Omega$ for S-16MFCs. In literature it has been reported that the change of the external resistance can cause a change in microorganisms diversity (Jung \& Regan, 2011) and that low external resistances favors the mass transport within the biofilm and the COD removal(Liu \& Tay, 2001) because of the formation of water channels (Zhang et al., 2011). These channels deteriorate the 
contact between microorganisms and anode decreasing the electrical performance (Zhang et al., 2011), which can be observed in fig. 10.

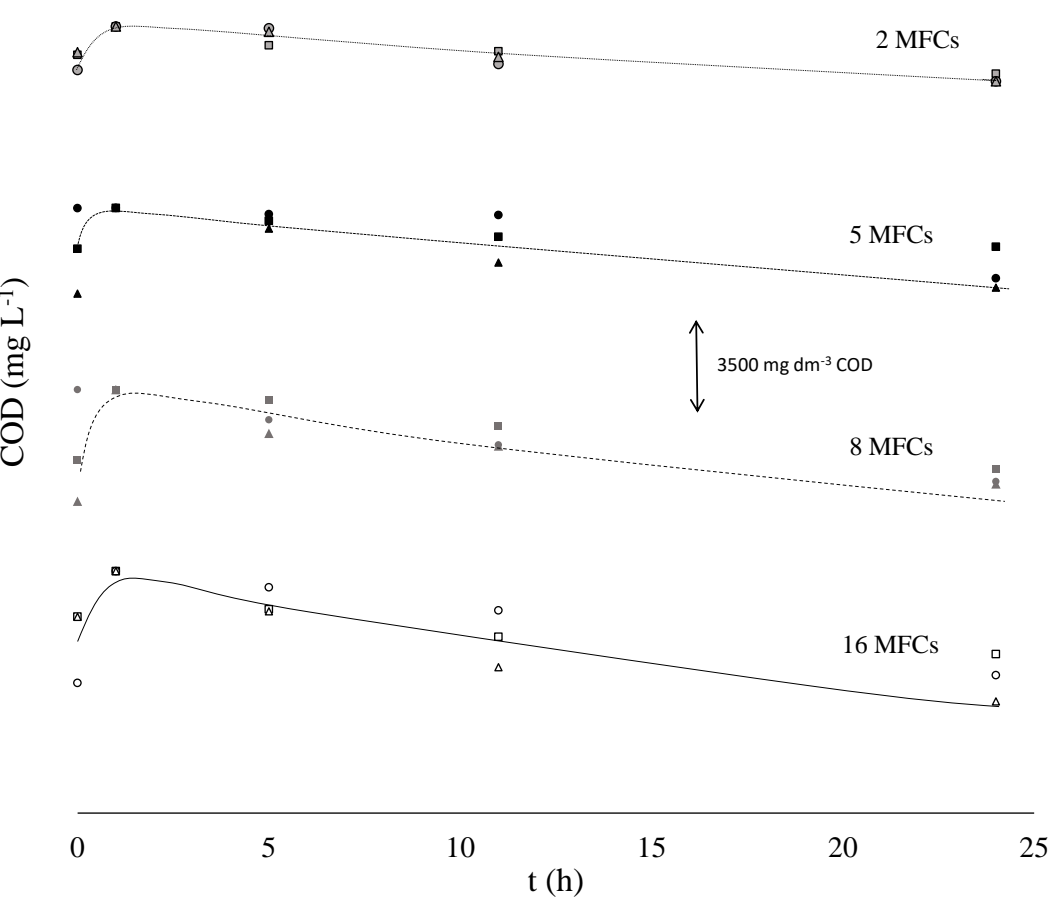

Figure 9. COD concentration evolution during each daily cycle with the kind of electrical connection: $(\square)$ single; $(\circ)$ parallel, $(\Delta)$ in series.

Despite the increase in the COD removal for the stack of 16 MFCs connected hydraulically to a sole reservoir tank, the electrical performance was not improved. In order to enhance its performance, it was proposed to feed each MFC with an individual tank instead of a sharing the same reservoir tank between all. A drastic improvement of the performances was obtained as shown in Fig. 10, that reports the power density curves obtained for the arrangements of MFCs in series and in parallel. Results demonstrate that individual feeding favors considerably the electrical performance of the stack regardless the type of connection. As there was COD availability in the stack when operating with a sole reservoir tank, this result may be explained in terms of the higher availability of bacteria accumulated in the reservoir fuel tanks capable to form the biofilm. 


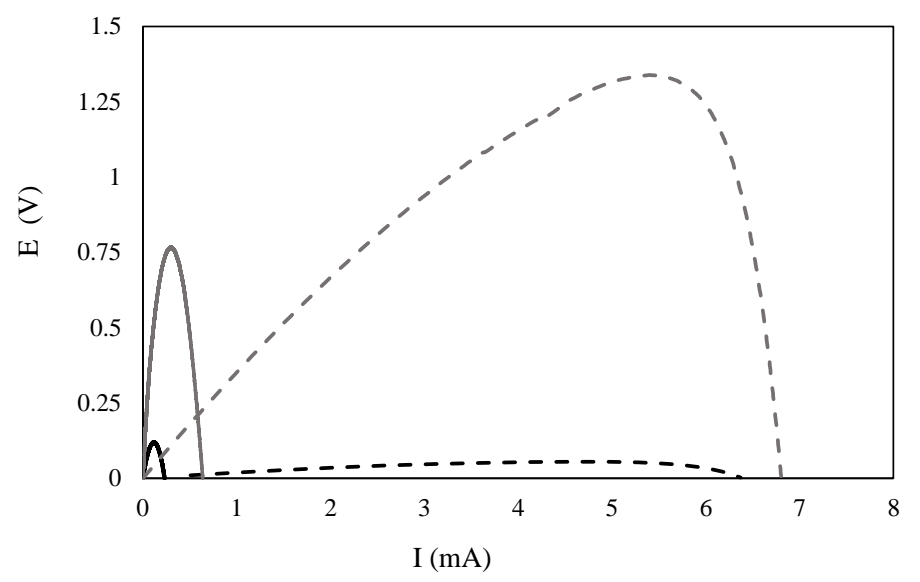

Figure 10. Power curves at the 16 MFCs stack steady state connected in parallel (dashedline) and in series (solid line) when the stack is fed by only one anodic reservoir (black line) and when an anodic reservoir is used per MFC of the stack (grey line).

At this point, it is important to evaluate the removal of COD. Thus, the one-MFC system removed $848 \mathrm{mg} \mathrm{L}^{-1}$ daily while the stack of 16 MFCs with a single fuel tank for all the units consumed $3516 \mathrm{mg} \mathrm{L}^{-1}$. When connecting a tank per MFC, the average removal for each tank of COD was $1350 \pm 462 \mathrm{mg} \mathrm{L}^{-1}$, which is very similar to the removal of the oneMFC system. In addition, the $\mathrm{pH}$ increase from 6.36 up to 7.35. These results can be an indicative of the development of a less electrogenic biofilms in the 16 MFCs stack fed by a unique reservoir and also points out the benefits of using an auxiliary tank per MFC instead of a reservoir per stack.

\section{Conclusions}

From this work, the following conclusions can be drawn:

- The higher the number of MFCs connected in a stack, the longer the period required for the steady-state electricity production. These longer time 
requirements are explained in terms of the larger available surface to form the biofilm in devices that were seeded with the same amount of sludge.

- The higher the number of MFCs fed with the same tank, the higher the concentration of COD removed. Slightly lower consumptions of COD were observed when the MFCs had individual electrical connections as compared to connections in series and parallel.

- The electricity production is limited in larger stacks by the volume of suspended solids available to form the biofilm. When a tank was used to feed all the stack, the current and power density output of the individual MFCs of the stack decreased with the number of MFCs arranged. The energy production was drastically enhanced when replacing the feeding system of a tank per stack by a tank per MFC.

- Stacking in series increased the cell potential of 6 times reaching $2.03 \mathrm{~V}$ with 8 MFCs while stacking in parallel resulted in a current of $6.98 \mathrm{~mA}, 4.2$ times more than that achieved with single connection. The power was increased up to 9.66 times stacking in series.

\section{Acknowledgements}

Authors are grateful for the financial support provided by the AEI (Agencia Estatal de Investigación) of the Spanish Ministry of Economy and Competitiveness (MINECO) through project CTQ2013-49748-EXP (Explora Program) and the Spanish Ministry of Education, Culture and Sport through the grant FPU 13/04118 of Sara Mateo.

\section{References}


Choi, S. 2015. Microscale microbial fuel cells: Advances and challenges. Biosensors and Bioelectronics, 69, 8-25.

Chouler, J., Padgett, G.A., Cameron, P.J., Preuss, K., Titirici, M.M., leropoulos, I., Di Lorenzo, M. 2016. Towards effective small scale microbial fuel cells for energy generation from urine. Electrochimica Acta, 192, 89-98.

D'Angelo, A., Mateo, S., Scialdone, O., Cañizares, P., Fernandez-Morales, F.J., Rodrigo, M.A. 2017. Optimization of the performance of an air-cathode MFC by changing solid retention time. Journal of Chemical Technology and Biotechnology.

Dekker, A., Ter Heijne, A., Saakes, M., Hamelers, H.V.M., Buisman, C.J.N. 2009. Analysis and improvement of a scaled-up and stacked microbial fuel cell. Environmental Science and Technology, 43(23), 9038-9042.

Federation, W.E., Association, A.P.H. 2005. Standard methods for the examination of water and wastewater. American Public Health Association (APHA): Washington, DC, USA.

Fernandez-Morales, F.J., Villasenor, J., Infantes, D. 2010. Modeling and monitoring of the acclimatization of conventional activated sludge to a biohydrogen producing culture by biokinetic control. International Journal of Hydrogen Energy, 35(20), 10927-10933.

leropoulos, I., Greenman, J., Melhuish, C. 2010. Improved energy output levels from small-scale Microbial Fuel Cells. Bioelectrochemistry, 78(1), 44-50.

leropoulos, I., Greenman, J., Melhuish, C. 2008. Microbial fuel cells based on carbon veil electrodes: Stack configuration and scalability. International Journal of Energy Research, 32(13), 1228-1240.

Ieropoulos, I., Greenman, J., Melhuish, C., Horsfield, I. EcoBot-III-A Robot with Guts. 2010. pp. 733-740.

leropoulos, I.A., Greenman, J., Melhuish, C. 2013a. Miniature microbial fuel cells and stacks for urine utilisation. International Journal of Hydrogen Energy, 38(1), 492-496.

leropoulos, I.A., Ledezma, P., Stinchcombe, A., Papaharalabos, G., Melhuish, C., Greenman, J. 2013b. Waste to real energy: the first MFC powered mobile phone. Physical Chemistry Chemical Physics, 15(37), 15312-15316.

Jung, S., Regan, J.M. 2011. Influence of external resistance on electrogenesis, methanogenesis, and anode prokaryotic communities in microbial fuel cells. Applied and environmental microbiology, 77(2), 564-571.

Kim, T., Kang, S., Kim, H.W., Paek, Y., Sung, J.H., Kim, Y.H., Jang, J.K. 2017. Assessment of organic removal in series-and parallel-connected microbial fuel cell stacks. Biotechnology and Bioprocess Engineering, 22(6), 739-747.

Ledezma, P., Greenman, J., leropoulos, I. 2013. MFC-cascade stacks maximise COD reduction and avoid voltage reversal under adverse conditions. Bioresource Technology, 134, 158165.

Liu, H., Ramnarayanan, R., Logan, B.E. 2004. Production of electricity during wastewater treatment using a single chamber microbial fuel cell. Environmental science \& technology, 38(7), 2281-2285.

Liu, Y., Tay, J.-H. 2001. Strategy for minimization of excess sludge production from the activated sludge process. Biotechnology advances, 19(2), 97-107.

Logan, B.E. 2008. Microbial fuel cells. John Wiley \& Sons.

Mateo, S., D'Angelo, A., Scialdone, O., Canizares, P., Andres Rodrigo, M., Jesus FernandezMorales, F. 2017a. The influence of sludge retention time on mixed culture microbial fuel cell start-ups. Biochemical Engineering Journal, 123, 38-44.

Mateo, S., Fernandez-Morales, F.J., Cañizares, P., Rodrigo, M.A. 2017b. Influence of the Cathode Platinum Loading and of the Implementation of Membranes on the Performance of AirBreathing Microbial Fuel Cells. Electrocatalysis, 1-8.

Mateo, S., Gonzalez del Campo, A., Lobato, J., Rodrigo, M., Canizares, P., Fernandez-Morales, F.J. 2016. Long-term effects of the transient COD concentration on the performance of microbial fuel cells. Biotechnology Progress, 32(4), 883-890. 
Mateo, S., Rodrigo, M., Fonseca, L.P., Cañizares, P., Fernandez-Morales, F.J. 2015a. Oxygen availability effect on the performance of air-breathing cathode microbial fuel cell. Biotechnology Progress, 31(4), 900-907.

Mateo, S., Rodrigo, M., Fonseca, L.P., Cañizares, P., Fernandez-Morales, F.J. 2015b. Oxygen availability effect on the performance of air-breathing cathode microbial fuel cell. Biotechnology Progress.

Mateo, S., Zamorano-López, N., Borras, L., Fernandez-Morales, F.J., Cañizares, P., Seco, A., Rodrigo, M. Effect of sludge age on microbial consortia developed in MFCs. Journal of Chemical Technology \& Biotechnology, n/a-n/a.

Mateo, S., Zamorano-López, N., Borras, L., Fernandez-Morales, F.J., Cañizares, P., Seco, A., Rodrigo, M. Effect of sludge age on microbial consortia developed in MFCs. Journal of Chemical Technology and Biotechnology.

Monasterio, S., Mascia, M., Di Lorenzo, M., Vacca, A., Palmas, S. 2015. A cascade of miniature microbial fuel cells coupled with an electrochemical reactor for energy harvesting. 6th European Fuel Cell Technology and Applications Conference - Piero Lunghi Conference, EFC 2015. ENEA. pp. 305-306.

Oliot, M., Etcheverry, L., Mosdale, R., Bergel, A. 2017. Microbial fuel cells connected in series in a common electrolyte underperform: Understanding why and in what context such a set-up can be applied. Electrochimica Acta, 246, 879-889.

Papaharalabos, G., Greenman, J., Melhuish, C., leropoulos, I. 2015. A novel small scale Microbial Fuel Cell design for increased electricity generation and waste water treatment. International Journal of Hydrogen Energy, 40(11), 4263-4268.

Qian, F., Morse, D.E. 2011. Miniaturizing microbial fuel cells. Trends in Biotechnology, 29(2), 6269.

Ringeisen, B.R., Henderson, E., Pietron, J.J., Little, B., Ray, R., Jones-Meehan, J. 2005. Aerobic power generation by a miniaturized microbial fuel cell. 230th ACS National Meeting, Washington, DC.

Ringeisen, B.R., Henderson, E., Wu, P.K., Pietron, J., Ray, R., Little, B., Biffinger, J.C., JonesMeehan, J.M. 2006. High power density from a miniature microbial fuel cell using Shewanella oneidensis DSP10. Environmental Science and Technology, 40(8), 26292634.

Ringeisen, B.R., Ray, R., Little, B. 2007. A miniature microbial fuel cell operating with an aerobic anode chamber. Journal of Power Sources, 165(2), 591-597.

Rodríguez Mayor, L., Villaseñor Camacho, J., Fernández Morales, F.J. 2004. Operational optimisation of pilot scale biological nutrient removal at the Ciudad Real (Spain) domestic wastewater treatment plant. Water, Air, and Soil Pollution, 152(1-4), 279-296.

Vicari, F., Mateo, S., Fernandez-Morales, F.J., Cañizares, P., Galia, A., Scialdone, O., Rodrigo, M.A. 2017. Influence of the methodology of inoculation in the performance of air-breathing microbial fuel cells. Journal of Electroanalytical Chemistry, 803, 81-88.

Walter, X.A., Forbes, S., Greenman, J., leropoulos, I.A. 2016a. From single MFC to cascade configuration: The relationship between size, hydraulic retention time and power density. Sustainable Energy Technologies and Assessments, 14, 74-79.

Walter, X.A., Gajda, I., Forbes, S., Winfield, J., Greenman, J., leropoulos, I. 2016b. Scaling-up of a novel, simplified MFC stack based on a self-stratifying urine column. Biotechnology for Biofuels, 9(1).

Water Environment, F., Water Pollution Control, F., American Water Works, A., American Public Health, A. 1905. Standard methods for the examination of water and wastewater. v.

Winfield, J., leropoulos, I., Greenman, J. 2012. Investigating a cascade of seven hydraulically connected microbial fuel cells. Bioresource Technology, 110, 245-250.

Winfield, J., leropoulos, I., Greenman, J., Dennis, J. 2011. Investigating the effects of fluidic connection between microbial fuel cells. Bioprocess and Biosystems Engineering, 34(4), 477-484. 
Zhang, L., Zhu, X., Li, J., Liao, Q., Ye, D. 2011. Biofilm formation and electricity generation of a microbial fuel cell started up under different external resistances. Journal of Power Sources, 196(15), 6029-6035.

Zhang, Y., Angelidaki, I. 2012. Self-stacked submersible microbial fuel cell (SSMFC) for improved remote power generation from lake sediments. Biosensors and Bioelectronics, 35(1), 265-270.

Zhao, N., Angelidaki, I., Zhang, Y. 2017. Electricity generation and microbial community in response to short-term changes in stack connection of self-stacked submersible microbial fuel cell powered by glycerol. Water Research, 109, 367-374. 